Available online at www.sciencedirect.com

science@direct.

Brain and Cognition $\mathrm{xxx}(2005) \mathrm{xxx}-\mathrm{xxx}$
Brain Cognition

www.elsevier.com/locate/b\&c

\title{
Crowded and sparse domains in object recognition: Consequences for categorization and naming
}

\author{
Tim M. Gale ${ }^{\mathrm{a}, \mathrm{b}, \mathrm{c}, *}$, Keith R. Laws $^{\mathrm{c}}$, Kerry Foley ${ }^{\mathrm{a}}$ \\ ${ }^{a}$ Department of Psychiatry, QEII Hospital, Welwyn Garden City, UK \\ ${ }^{\mathrm{b}}$ School of Computer Science, University of Hertfordshire, Hatfield, UK \\ ${ }^{\mathrm{c}}$ School of Psychology, University of Hertfordshire, Hatfield, UK
}

Accepted 24 October 2005

\begin{abstract}
Some models of object recognition propose that items from structurally crowded categories (e.g., living things) permit faster access to superordinate semantic information than structurally dissimilar categories (e.g., nonliving things), but slower access to individual object information when naming items. We present four experiments that utilize the same matched stimuli: two examine superordinate categorization and two examine picture naming. Experiments 1 and 2 required participants to sort pictures into their appropriate superordinate categories and both revealed faster categorization for living than nonliving things. Nonetheless, the living thing superiority disappeared when the atypical categories of body parts and musical instruments were excluded. Experiment 3 examined naming latency and found no difference between living and nonliving things. This finding was replicated in Experiment 4 where the same items were presented in different formats (e.g., color and line-drawn versions). Taken as a whole, these experiments show that the ease with which people categorize items maps strongly onto the ease with which they name them.
\end{abstract}

(C) 2005 Published by Elsevier Inc.

Keywords: Category-specific; Visual crowding; Perceptual differentiation; Picture naming; Picture categorization

\section{Introduction}

Understanding and interpreting category-specific impairments in patients relies upon knowing how a neurologically intact population performs on the same kinds of task; and this has, until relatively recently, been neglected in most accounts of category specificity (see Laws, in press; Laws, Gale, Leeson, \& Crawford, 2005). Given the greater frequency of living thing impairments reported to date it has often been assumed that normal controls should be less accurate and slower to name items from living thing categories. Indeed, some studies have described this pattern and the explanation given is that living things share greater intra-category structural similarity relative to nonliving

\footnotetext{
* Corresponding author. Fax: +44 01707365169 .

E-mail address: t.gale@herts.ac.uk (T.M. Gale).
}

things (Gaffan \& Heywood, 1993; Humphreys, Riddoch, \& Quinlan, 1988; Lloyd-Jones \& Humphreys, 1997a, 1997b).

Some models of object recognition assume that competition between structural descriptions gives rise to processing advantages for certain classes of stimuli (Gerlach, 2001; Humphreys \& Forde, 2001; Humphreys et al., 1988; Tranel, Logan, Frank, \& Damasio, 1997). Furthermore, models such as the Cascade (Humphreys et al., 1988) and Hierarchical Interactive Theory (HIT: Humphreys \& Forde, 2001) propose that the direction of category advantage depends upon the level of processing required by a task. For example, if a target item shares a similar structural description to several within-category associates, it will take longer to resolve a specific 'structural' representation than if the target item is structurally distinctive. This will affect the activation of item-specific semantic and phonological representations such that a significant delay in object naming should be measurable. But, by contrast, greater competition for some categories (e.g., living things) at the level of 
structural representation should produce a processing advantage on tasks where exactly the same stimuli require superordinate category recognition. Presumably, if living things are characterized by greater structural similarity, those visual properties that are common to superordinate category members should be readily available and therefore promote easier superordinate categorization for this class of items. By contrast, the very same properties that coherently unite items from living thing superordinates will make them more difficult to discriminate at the item level.

Few studies have compared superordinate categorization between living and nonliving things, and the evidence in favor of the proposals outlined above is somewhat equivocal (Lloyd-Jones \& Humphreys, 1997b; Price \& Humphreys, 1989). Notably, one reports faster naming and classification for living (structurally similar) things (LloydJones \& Humphreys, 1997b); while the other reports no difference for naming, but a living advantage for classification (Price \& Humphreys, 1989). Furthermore, Price and Humphreys (1989) did not systematically control for variables such as word frequency, visual complexity, and concept familiarity (all of which are known to disadvantage the naming of living things). Lloyd-Jones and Humphreys (1997b) co-varied these nuisance variables, but they examined a very restricted range of items, e.g., fruit and vegetables versus clothing and furniture. More recent studies have shown that when the above named variables are matched across category, normal subjects tend to be more accurate and faster to name living things (Brousseau \& Buchanan, 2004; Laws, 1999, 2000; Laws \& Gale, 2002; Laws, Leeson, \& Gale, 2002; Laws \& Neve, 1999; McKenna \& Parry, 1994). Hence, the data on these issues remain uncertain.

Another important issue concerns the treatment of categories that appear to elicit counter-intuitive response profiles in patients. Many studies have noted that musical instruments tend to associate with living things while body parts seem to behave more like nonliving things (Barbarotto, Capitani, \& Laiacona, 2001; Laws, Gale, Frank, \& Davey, 2002; Parkin \& Stewart, 1993). It is therefore important to examine the contribution of these categories to any emergent category effects. In this paper, we present four experiments which examine superordinate categorization and picture naming using the same sets of stimuli across each task. If living things are more visually crowded, we should predict a double dissociation in categories across tasks.

\section{Experiment 1}

\subsection{Method}

\subsubsection{Participants}

Fifty participants were recruited ( 25 male, 25 female) with a mean age of $33( \pm 14)$ years. All had normal or corrected-to-normal vision, none had cognitive or perceptual impairments, and all spoke English as their first language. The group comprised hospital administrative and cleaning staff, nurses and students.

\subsubsection{Materials}

107

We used 100 picture cards depicting items from 10 different superordinate categories. There were five living thing categories (animals, birds, body parts, fruit, and vegetables) and five nonliving thing categories (clothing, furniture, musical items, tools, and vehicles), with 10 different items per category. The pictures were grey scale versions of the Snodgrass and Vanderwart (1980) corpus adapted by Rossion and Pourtois (2004). These pictures use the same line detail as the original corpus, but also include grey scale shading and some textural detail, thereby providing representations that are more realistic. The pictures were presented on laminated cards of $10 \mathrm{~cm}^{2}$. A full list of items appears in Appendix A.

Living and nonliving things were matched for, familiarity $(3.24 \pm 1.01$ vs. $3.53 \pm 0.87, F[1,98]=2.41, p>.1)$, visual complexity $(3.01 \pm 0.93$ vs. $3.03 \pm 0.85, F[1,98]<1, p>.9)$ from Snodgrass and Vanderwart (1980), and log word frequency $(1.11 \pm 0.64$ vs. $1.13 \pm 0.75, F[1,98]<1, p=.88$; from Kuçera \& Francis, 1967).

\subsubsection{Procedure}

The picture cards were divided into two randomly shuffled sets containing the 50 living and the 50 nonliving things. The aim of the task was to sort each pack into its five superordinate categories as quickly as possible. This was done by placing the cards underneath 5 large category labels (e.g., "animals," "fruit," etc.). The first pack of 50 cards was laid facedown in front of the participant who then turned one card over at a time and allocated it to the appropriate category label. If a card was accidentally allocated to the wrong category, the participant was allowed to correct the mistake. The time required to complete the task (i.e., to sort all 50 cards into their appropriate categories) was recorded by stopwatch from the moment that the first card was turned over to the time at which the last card was allocated. Each participant sorted both packs of cards but the task order (L, NL vs. NL, L) was randomly determined for each participant.

\subsection{Results and summary}

\subsubsection{Errors and outliers}

The number of sorting errors was low (the means for living and nonliving things were 2.44 and $2.48 \%$, respectively, $F[1,49]<1, p>9)$. The breakdown of errors by superordinate category was as follows: birds $(6.4 \%)$, vegetables $(5.4 \%)$, furniture $(5 \%)$, vehicles $(4.8 \%)$, musical Items $(2 \%)$, tools $(0.6 \%)$, fruit $(0.4 \%)$, clothing $(0 \%)$, body parts $(0 \%)$, and animals $(0 \%)$. Sorting times exceeding two standard deviations above or below the mean were removed (this resulted in two being removed for living things and three for nonliving things).

\subsubsection{Category differences}

Living things were sorted into their five superordinate categories significantly faster than nonliving things $(94.9 \pm 17.8$ vs. $101.5 \pm 15$ s: $F[1,93]=13.06, p<.001)$. 
160 This finding accords with the notion that greater visual 161 crowding facilitates the identification of living than nonliv162 ing thing category members. The task used in Experiment 1 163 does not, however, address the question of whether any rel164 ative difficulty exists across the 10 categories. For example, 165 do some superordinate categories produce atypical profiles 166 that might mask or distort the overall living or nonliving 167 profile? We turn to this in Experiment 2.

\section{Experiment 2}

\subsection{Method}

\subsubsection{Participants}

Seventy-eight participants were recruited (39 female, 39 male) with a mean age of $36( \pm 12.4)$ years. All had normal or corrected-to-normal vision, none had cognitive or perceptual impairments, and all spoke English as their first language. The group comprised postgraduate engineering and computing students, department store workers, and hospital administrative staff.

\subsubsection{Materials}

The materials were identical to those described in Experiment 1 .

\subsubsection{Procedure}

In this experiment, participants selected items belonging to a target superordinate category from an array of distractors. Each trial comprised 30 picture cards representing three superordinate categories (e.g., animals, vegetables, furniture). These were shuffled and laid out on the desktop in an array of six columns by five rows. The array was covered until the participant was ready to begin the trial. The participant was then given a set of 10 large $(3 \mathrm{~cm}$ in diameter) identical coins that were numbered consecutively from 1 to 10 . The participant was asked to identify all 10 items belonging to the given target category (e.g., animals) by placing the coins on the appropriate picture cards within the array. This method of marking the target cards was found to be more reliable than either (i) picking the cards up by hand or (ii) sorting them into target and nontarget piles. The latter approaches have been used in previous studies, but may create difficulties for subjects (for example, if participants have trouble in picking up some of the cards, or drop them).

The time taken to complete the trial was recorded in seconds and milliseconds from the moment the covering was removed until the last coin had been placed. The order in which items were identified as target category members for each trial was recorded by noting the number of the coin (1-10) that was placed on each picture. Each participant completed three trials, one after the other [i.e., they saw three different arrays comprising 90 cards ( 9 categories) in total]. None of the individual cards or categories was seen more than once by any participant. A running order of target and distractor categories was drawn up in advance to ensure that each category appeared as a target an equal number of times across all trials. Moreover, each of the 10 categories appeared as distractors with equal probability. In each array of 30 cards, the two distractor categories always comprised one living and one nonliving category.

\subsection{Results and summary}

\subsubsection{Errors and outliers}

A total of 234 trials were run. Categorization errors, where a participant placed a coin on a nontarget category item and did not correct the error, were made in only 15 $(6.4 \%)$ trials and these were excluded from the latency analyses. These errors were distributed as follows: musical items (5), birds (4), fruit (2), vegetables (2), and furniture (2). Of the 219 remaining trials, a further 11 were also excluded because the latencies exceeded two standard deviations beyond the mean. These were exclusive cases, where participants spent a lot of time pondering on whether a specific item should be included in a category or not (one example being whether 'piano' should be classed as a musical instrument or an item of furniture). All primary analyses were run with outliers included and excluded. Excluding them made no difference to the statistical significance of any of the tests. However, the descriptive and inferential statistics we report here are based on the smaller dataset of 208 trials since this represents the total number of trials completed accurately and without distraction.

\subsubsection{Category differences}

The mean time for selecting items from living thing categories was $12.90( \pm 4.32) \mathrm{s}$ compared with $14.61( \pm 4.60) \mathrm{s}$ for nonliving things. This difference was reliable $(F[1,206]=7.62, p<.01)$. This difference, however, disappeared when the categories of body parts and musical instruments were removed from the analysis (13.49 vs. 13.95: $F[1,168]<1)$. With body parts removed, the means for living vs. nonliving were $13.31( \pm 4.7)$ vs. $14.61( \pm 4.6)$, $F[1,186]=3.64, p=.06$. With musical instruments removed, the means for living vs. nonliving were $12.90( \pm 4.3)$ vs. 13.89 $( \pm 4.2), F[1,189]=2.54, p=.11$. A breakdown of mean RTs by superordinate category is displayed in Table 1 .

Table 1

Mean and SD latencies for the 10 categories, along with the mean values on background variables (in Experiment 2)

\begin{tabular}{lllll}
\hline Category & Mean RT [SD] & Familiarity & VC & WF $(\log )$ \\
\hline Animals & $10.67[2.86]$ & 2.63 & 3.85 & 1.26 \\
Body parts & $11.23[1.39]$ & 4.67 & 2.38 & 1.79 \\
Clothing & $11.76[2.52]$ & 4.11 & 2.54 & 1.43 \\
Birds & $12.93[5.21]$ & 2.29 & 3.43 & 0.83 \\
Fruit & $13.50[3.61]$ & 3.39 & 2.31 & 0.89 \\
Tools & $13.71[4.64]$ & 3.1 & 2.36 & 0.71 \\
Furniture & $14.88[3.83]$ & 4.27 & 2.62 & 1.45 \\
Vehicle & $15.44[4.82]$ & 3.62 & 3.73 & 1.16 \\
Vegetable & $16.86[4.93]$ & 3.22 & 3.1 & 0.58 \\
Musical items & $17.89[5.01]$ & 2.56 & 3.89 & 0.84 \\
\hline
\end{tabular}


Table 2

The range of mean retrieval positions for items in the 10 superordinates

\begin{tabular}{llll}
\hline Category & $\begin{array}{l}\text { Range of mean retrieval } \\
\text { position across 10 exemplars }\end{array}$ & $\begin{array}{l}\text { Most common } \\
\text { first selection }\end{array}$ & $\begin{array}{l}\text { Most common } \\
\text { last selection }\end{array}$ \\
\hline Animals & $4.04-6.17$ & Cow & Goat \\
Body Parts & $4.86-6.52$ & Toe & Arm \\
Clothing & $4.09-7.00$ & Sweater & Shoe \\
Birds & $4.23-6.73$ & Rooster & Peacock \\
Fruit & $4.09-7.36$ & Strawberry & Banana \\
Tools & $3.96-7.23$ & Wrench & Paintbrush \\
Furniture & $4.10-7.70$ & Table & Television \\
Vehicle & $4.14-6.76$ & Bicycle & Sailboat \\
Vegetable & $3.82-7.00$ & Celery & Mushroom \\
Music & $4.32-6.82$ & Guitar & Accordion \\
\hline
\end{tabular}

A larger range is indicative of greater systematicity in item retrieval order (in Experiment 2). typical nonliving things are classified more quickly than atypical nonliving things; however, typicality makes no difference for living things. In other words, typicality determines the time taken to access semantic information for nonliving, but not living things. For living things, it may be that categorization is determined more by structural information than semantic (typicality) information. In Experiment 3, we investigate object naming using exactly the same stimuli and examine whether the reverse dissociation is observed (i.e., a significant advantage for nonliving things).

\section{Experiment 3}

\subsection{Method}

\subsubsection{Participants}

Twenty undergraduate students (9 females, 11 males) of mean age 26 years (range 19-43) viewed all 100 pictures in a naming latency task. All had normal, or corrected-to-normal, vision and spoke English as their first language.

\subsubsection{Materials}

The same 100 grey scale pictures used in Experiment 1 were presented digitally.

\subsubsection{Procedure}

The stimuli were presented against a white background on a high-resolution monitor using SuperLab software run on an Apple Macintosh computer. Each drawing extended maximum dimensions of $9.91 \times 6.95 \mathrm{~cm}(281 \times 197$ pixels $)$ and was viewed from a distance of $50 \mathrm{~cm}$. There was no time limit for responding. Participants were asked to name each item as it appeared on the screen and the latency of their response was recorded using a voice key. A blank white screen appeared between each presented picture for $1000 \mathrm{~ms}$. Pictures were presented randomly and timing accuracy was to within one thousandth of a second. Before collating the raw latency data, any score that was three or more standard deviations beyond an individual participant's mean score were excluded, as was the latency for any item that was named incorrectly.

\subsection{Results and summary}

The naming error rate was very low $(0.3 \%$ of all responses) so we did not analyze errors. Three items generated mean latencies that exceeded two standard deviations beyond the pooled mean (i.e., they exceeded a cut-off score $\geqslant 1433 \mathrm{~ms}$ in these data). These items were: artichoke (1621 ms), asparagus $(1617 \mathrm{~ms})$, and French horn (1634 ms). Excluding these outlying items made no difference to the living vs. nonliving comparison. Comparison of naming latencies for living and nonliving things $(1022 \pm 158 \mathrm{~ms}$ vs. $1062 \pm 162 \mathrm{~ms})$ revealed no significant difference $(F[1,98]$ $=1.46, p=.23)$. Removing body parts and/or musical instruments made no difference to the results $(F<1)$.
304 


\subsubsection{Relationship between naming latency and superordinate} categorization latency

The mean naming latencies from Table 3 correlated significantly with the mean superordinate categorization latencies in Table $1(r=0.84, F[1,9]=18.6, p<.003)$. Animals, body parts and clothing, the three categories with the quickest superordinate categorization times, were also the fastest named categories. Similarly, musical instruments and vegetables elicited the two slowest latencies on both the superordinate categorization and item naming tasks.

If the apparent advantages for living thing categorization in Experiments 1 and 2 reflect greater visual overlap for living things, we would have expected the reverse dissociation on the same set of stimuli in Experiment 3; however, no significant difference emerged in the naming latencies for living and nonliving things.

\section{Experiment 4}

Given the null finding in Experiment 3, we tested a new group of participants on the same 100 items, but this time depicted: as black and white line drawings (as per the original Snodgrass and Vanderwart corpus); and as color drawings (also recently adapted and published by Rossion \& Pourtois, 2004).

\subsection{Method}

\subsubsection{Participants}

Thirty undergraduate students (18 females, 12 males) of mean age 25 years (range 18-48) viewed the monochrome and color pictures. All participants had normal, or corrected-to-normal, vision and spoke English as their first language.

\subsubsection{Materials}

The same 100 items used in Experiments 1-3 were presented digitally as (i) monochrome line drawings and (ii) color versions for a naming latency task.

\subsubsection{Procedure}

The procedure was identical to that of Experiment 3.

Table 3

Mean picture-naming latencies by superordinate category (in Experiment 3)

\begin{tabular}{lclll}
\hline Category & $\begin{array}{l}\text { Mean } \\
\text { naming RT }\end{array}$ & $\begin{array}{l}\text { Range } \\
\text { (means) }\end{array}$ & $\begin{array}{l}\text { Fastest } \\
\text { named }\end{array}$ & $\begin{array}{l}\text { Slowest } \\
\text { named }\end{array}$ \\
\hline Body parts & 899 & $761-1044$ & Eye & Finger \\
Animals & 1010 & $784-1114$ & Dog & Cow \\
Clothing & 1028 & $732-1381$ & Sock & Coat \\
Vehicle & 1042 & $825-1415$ & Airplane & Wagon \\
Tools & 1048 & $826-1281$ & Saw & Pliers \\
Birds & 1068 & $781-1359$ & Owl & Chicken \\
Fruit & 1072 & $887-1269$ & Pear & Orange \\
Furniture & 1079 & $899-1421$ & Television & Desk \\
Musical items & 1167 & $885-1634$ & Bell & French horn \\
Vegetables & 1189 & $821-1621$ & Carrot & Artichoke \\
\hline
\end{tabular}

5.2. Results summary

(i) Monochrome drawings (BW): living and nonliving things $(978 \pm 172 \mathrm{~ms}$ vs. $1017 \pm 142 \mathrm{~ms})$ revealed no significant difference in naming latency $(F[1,98]=$ $1.54, p=.22$ ). Removing body parts and/or musical instruments from the analysis made no difference to the results $(F<1)$. Naming errors constituted less than $1 \%$ of total responses and were not analyzed.

(ii) Color pictures: living and nonliving things ( $945 \pm 165 \mathrm{~ms}$ vs. $953 \pm 178 \mathrm{~ms}$ ) again failed to reveal any significant difference $(F[1,98]<1)$. Removing body parts and/or musical instruments from the analysis made no difference to the results $(F<1)$. Again, the naming error rate was less than $1 \%$.

Experiment 4 again did not find the predicted advantage for nonliving naming. These results confirmed those of Experiment 3, and extended the findings to three variants of the same stimuli using different participant groups.

\section{Discussion}

As far as we are aware, these experiments are the first to directly compare categorization and naming across category on the same set of matched stimuli from a broad range of categories. In this respect, this study directly examines hypotheses derived from models that emphasize a role for visual crowding (e.g., in particular, the notion that high structural similarity gives rise to more efficient access to super-ordinate semantic information and slowed access to information about individual objects).

In Experiments 1 and 2, participants selected items belonging to a target superordinate from an array of distractor items and were significantly faster to categorize items within living thing superordinates. Although this pattern is consistent with the assumptions and predictions of visual crowding models, the latency difference disappeared when the atypical living and nonliving categories of body parts and musical instruments were excluded (Experiment 2). Additionally, Experiments 3 and 4 failed to reveal a picture naming advantage in favor of nonliving things (as predicted by visual crowding models). Again, body parts and musical instruments were among the fastest and slowest, respectively, to be named. Hence, the treatment of these two superordinates may play a critical role in determining the outcomes of category-specific studies.

The proposal that naming should be faster for nonliving (structurally dissimilar) than living (structurally similar) things failed to receive any support from the current study, with no difference emerging on the purportedly more sensitive task of picture naming (i.e., where more specific and uniquely identifying information is required). Of course, with null results it is possible that the study did not have sufficient power to detect a true difference. We calculated the $95 \%$ confidence intervals for the living-nonliving effect size for naming grey scale, monochrome and color images 
$(d=0.24, \mathrm{CI}=-0.15$ to $0.63 ; d=0.25, \mathrm{CI}=-0.15$ to $0.64 ;$ $d=0.14, \mathrm{CI}=-0.25$ to 0.53 , respectively). ${ }^{1}$ As expected from the nonsignificant results on all stimulus sets, the confidence intervals span through zero. In each case, the effect size is small and is in the direction of an advantage for naming living things (which runs contrary to the position of some authors). Looking at the confidence intervals for the effect sizes, it is clear that at best, the effect size in favor of faster naming of nonliving relative to living things is likely to be very small $(-0.15,-0.15$, and -0.25$)$. The replication of a null result using three varieties of the same items leads us to believe that this is a genuine finding; and cannot be attributed to confounds such as familiarity, visual complexity, name frequency or age-of-acquisition. Leaving aside the lack of evidence for nonliving naming, we also need to consider why the data presented here also fail to accord with studies that report a living advantage (Brousseau \& Buchanan, 2004; Laws, 1999, 2000; Laws \& Gale, 2002; Laws et al., 2002; Laws \& Neve, 1999; McKenna \& Parry, 1994). One possibility is that, under normal viewing conditions, any effect size is small for picture naming and that small changes in stimuli or presentation and response conditions may affect the living advantage. Recent studies with normal healthy subjects seem to support the latter notion (Gerlach, 2001; Låg, in press). Nonetheless, the direction of the findings in Experiments 3 and 4 were always in the opposite direction to that predicted by visual crowding hypotheses (i.e., living things were named slightly faster). Whatever the reason for the living advantage appearing or disappearing in some studies, little evidence supports a nonliving naming advantage when matched stimuli are presented in normal viewing conditions.

The data presented in this paper suggest several things. First, explanations for category specific deficits must address naming and categorization performance for atypical categories. Second, that within the context of atypical categories, the profile seen in patients is mirrored by that found in neurologically intact individuals and so may be an exaggeration of the normal profile (i.e., body parts being relatively preserved and musical instruments being poorly recognized and named). Finally, although the structural similarity hypothesis suggests that superordinate picture classification and picture naming should provide inverse profiles, our categorization and naming experiments show that the ease with which people classify items maps strongly onto the ease with which they name them, i.e., the same categories that are difficult or easy to classify are difficult/easy to name.

\footnotetext{
1 To address the possibility raised by one reviewer, that subjects were producing low error rates at the cost of generally slowed latencies (at a ceiling), we compared just the fastest $50 \%$ of living and nonliving latencies for the three picture sets. Each was matched for familiarity, name frequency and visual complexity across category. No significant differences emerged for category and in each case living things were again named more quickly.
}

\section{Acknowledgments}

We thank Prof. Glyn Humphreys, Prof. Henri Cohen, and an anonymous reviewer for their helpful comments on earlier drafts of this paper. We are also very grateful to Verity Leeson for her assistance in collecting naming latency data.

Appendix A. List of items used in all experiments

ANIMAL
BEAR
COW
DOG
ELEPHANT
GIRAFFE
GOAT
HORSE
LION
SHEEP
SQUIRREL
BIRD
CHICKEN
DUCK
EAGLE
OSTRICH
OWL
PEACOCK
PENGUIN
ROOSTER
SWAN
BODY PART
ARM
EAR
EYE
FINGER
FOOT
HAND
LEG
LIPS
NOSE
TOE
CLOTHING
COAT
DRESS
HAT
JACKET
PANTS
SHIRT
SHOE
SKIRT
SOCK
SWEATER
FRUIT
APPLE
BANANA
CHERRY
GRAPES
LEMON
ORANGE
PEACH
PEAR




\section{PINEAPPLE}

STRAWBERRY

FURNITURE

BED

CHAIR

$\mathrm{COUCH}$

DESK

DRESSER

REFRIDGERATOR

ROCKING CHAIR

STOOL

TABLE

TELEVSION

MUSICAL INSTRUMENT

ACCORDIAN

BELL

DRUM

FLUTE

FRENCH HORN

GUITAR

HARP

PIANO

TRUMPET

VIOLIN

TOOL

AXE

CHISEL

HAMMER

PAINTBRUSH

PLIERS

RULER

SAW

SCISSORS

SCREWDRIVER

WRENCH

VEGETABLE

ARTICHOKE

ASPARAGUS

CARROT

CELERY

LETTUCE

MUSHROOM

ONION

PEPPER

POTATO

PUMPKIN

VEHICLE

AEROPLANE

BIKE

BUS

CAR

HELICOPTER

MOTORBIKE

SAILBOAT

TRAIN

TRUCK

WAGON

References
Battig, W. F., \& Montague, W. E. (1969). Category norms for verbal items in 56 categories: A replication and extension of the Connecticut category norms. Journal of Experimental Psychology, 80, 1-146.

Brousseau, G., \& Buchanan, L. (2004). Semantic category effect and emotional valence in female university students. Brain and Language, 90, 241-248.

Gaffan, D., \& Heywood, C. A. (1993). A spurious category-specific visual agnosia for living things in normal human and non-human primates. Journal of Cognitive Neuroscience, 5, 118-128.

Gerlach, C. (2001). Structural similarity causes different category effects depending on task characteristics. Neuropsycholgia, 39, 895-900.

Humphreys, G. W., \& Forde, E. M. E. (2001). Hierarchies, similarity and interactivity in object recognition: On the multiplicity of 'category-specific' deficits in neuropsychological populations. Behavioral and Brain Sciences, 24, 453-509.

Humphreys, G. W., Riddoch, J., \& Quinlan, P. T. (1988). Cascade processes in picture identification. Cognitive Neuropsychology, 5, 67-103.

Kuçera, H., \& Francis, W. (1967). Computational analysis of present-day American English Providence. Rhode Island: Brown University Press.

Låg, T. (in press). Category-specific effects in object identification: What is 'normal'? Cortex.

Laws, K. R. (1999). Gender affects naming latencies for living and nonliving things: Implications for familiarity. Cortex, 35, 729-733.

Laws, K. R. (2000). Category-specific naming errors in normal subjects: the influence of evolution and experience. Brain and Language, 75, 123 133

Laws, K. R. (in press). "Illusions of normality": A methodological critique of category-specific naming. Cortex.

Laws, K. R., \& Gale, T. M. (2002). Category-specific naming and the 'visual' characteristics of line drawn stimuli. Cortex, 38, 7-21.

Laws, K. R., Gale, T. M., Frank, R., \& Davey, N. (2002). Visual similarity is greater for line drawings of nonliving than living things: The importance of musical instruments and body-parts. Brain and Cognition, 48, $421-423$.

Laws, K. R., Gale, T. M., Leeson, V. C., \& Crawford, J. (2005). When is category specific in Alzheimer's disease? Cortex, 41, 452-463.

Laws, K. R., Leeson, V. C., \& Gale, T. M. (2002). The effect of 'masking' on picture naming latencies. Cortex, 38, 137-147.

Laws, K. R., \& Neve, C. A. (1999). 'Normal' category-specific advantage for naming living things. Neuropsychologia, 37, 1263-1269.

Lloyd-Jones, T., \& Humphreys, G. W. (1997a). Perceptual differentiation as a source of category effects in object processing: Evidence from naming and object decision. Memory and Cognition, 25, 18-35.

Lloyd-Jones, T., \& Humphreys, G. W. (1997b). Categorizing chairs and naming pears: category differences in object processing as a function of task and priming. Memory and Cognition, 25, 606-624.

McKenna, P., \& Parry, R. (1994). Category-specificity in the naming of natural and man-made objects. Neuropsychological Rehabilitation, 4, 255-281.

Parkin, A. J., \& Stewart, F. (1993). Category-specific impairments? No. A critique of Sartori et al. Quarterly Journal of Experimental Psychology, $46 A, 505-509$.

Price, C. J., \& Humphreys, G. W. (1989). The effects of surface detail on object categorization and naming. Quarterly Journal of Experimental Psychology, 41A, 797-828.

Rossion, B., \& Pourtois, G. (2004). Revisiting Snodgrass and Vanderwart's object pictorial set: the role of surface detail in basic-level object recognition. Perception, 33, 217-236.

Snodgrass, J. G., \& Vanderwart, M. (1980). A standardised set of 260 pictures: Norms for name agreement, image agreement, familiarity and visual complexity. Journal of Experimental Psychology: Human Learning and Memory, 6, 174-215.

Tranel, D., Logan, C. G., Frank, R. J., \& Damasio, A. R. (1997). Explaining category-related effects in the retrieval of conceptual and lexical knowledge for concrete entities: Operationalisation and analysis of factors. Neuropsychologia, 35, 1329-1339.
490 ments and inanimate body parts. Neuropsychologia, 39, 406-414.
metto 